\title{
Fishing for a diagnosis
}

\author{
NG Dewhurst \\ President of the Royal College of Physicians of Edinburgh and Consultant Cardiologist, Perth Royal Infirmary, UK
}

KEYWORDS Diagnosis, imaging, incidentaloma, investigation, phebotomy, rule-out

DECLARATION OF INTERESTS No conflict of interests declared.

\author{
Correspondence to NG Dewhurst, \\ Royal College of Physicians of \\ Edinburgh, 9 Queen Street, \\ Edinburgh EH2 IJQ
}

tel. +44 (0) I 3 I 2473638

e-mail n.dewhurst@rcpe.ac.uk
As a trainee physician I was introduced to the concept of two contrasting investigation methodologies, namely the use of the 'fishing rod' versus the 'casting of the net'. The first was highly selective and used a limited number of investigations focused on the probable diagnosis. The latter was reserved for the 'fishing rod failures' and only implemented in exceptional cases. With fewer investigations at our disposal and through assiduous gatekeeping by hard-pressed on-call laboratory staff, the initial investigation of the acute medical admission was historically and typically a selective and methodical process, with the net being cast only when diagnoses remained elusive. The piscatorial analogy is as valid now as it was then.

Changes in the intervening years have been dramatic. We are now able to access a much wider range of laboratory investigations, available 24/7 and with a faster results service into the bargain. We have national, regional and local guidelines in place to direct the trainees' selection of blood tests, the results of which assist in decision-making within time constraints to meet the appropriate deadlines. So far so good. But, as a result, there must be concern regarding the resulting changes in medical practice. I can anticipate being on call in the nottoo-distant future when all my patients admitted acutely will have had C-reactive protein measured routinely, many will be reassured that their magnesium levels are in fact normal and some will be subjected to repeat phlebotomy because the apparently standard coagulation screen or assay for D-dimers had been omitted. Some may feel I exaggerate, but many of you will already be familiar with this scenario. Within an hour or two of a patient now crossing the admissions unit threshold, the aforementioned net has been well and truly cast.

Do we not need to raise the awareness of trainees and students that the tick-box approach to requesting numerous blood investigations carries its own benefit/ risk ratio? Although important morbidities are promptly identified, performing superfluous blood investigations and repeating those tests with borderline abnormality has been implicated as a potential cause of the hospitalacquired anaemia described in all age groups.' 'Ticking the boxes' also impacts on our processes of diagnostic reasoning and how we approach the reporting and the significance of tests, be they positive or otherwise. For example, 'troponin-negative chest pain' now commonly features in referral letters I receive. It is as if the description is thought of as a valid diagnosis in its own right. At the same time it is a label that can be interpreted as being dismissive as an entity and therefore a patient not worthy of further investigation, even though the evidence is to the contrary. ${ }^{2}$

The twin-edged sword of medical investigation is not, alas, confined to blood analysis. Our investigational imaging menu has become similarly more expansive and available. The choices include not only more sophisticated computerised tomography (CT) but also magnetic resonance and ultrasound. With every new technological breakthrough in imaging, the subsequent media coverage and internet commentary heighten not only the awareness of the public but also, potentially, their anxiety. The expectations of both patient and primary-care physician are often evident at the time of assessment of the acute presentation. To compound the situation, an explanation of 'negative' tests is often challenging and time-consuming. For example, despite normal coronary angiograms in the context of apparently typical angina, the patient often remains symptomatic and the pathway to multiple serial investigations beckons. Timely re-evaluation of all aspects of the patient's history (including the potential stresses of modern-day living) could prevent what would otherwise be the knee-jerk referral for upper gastrointestinal studies.

Fast-track CT imaging from emergency departments rightly features in many current diagnostic pathways and protocols. In an era of the practice of defensive medicine and with increasing access to multidetector scanners, the 'triple rule-out' examination is destined to become the norm for patients presenting with acute chest pain. The use of the negative 'rule-out', however, already anticipates the relatively low pick-up rate for the acute coronary disease, aortic dissection or pulmonary embolism for which we were searching in the first place. ${ }^{3}$ Indeed, with vascular calcification so common after middle age, its presence in the coronary circulation may be coincidental rather than causative in the context of the presentation. To complicate things further, vulnerable plaque may neither be calcified nor flow-limiting before the final rupture causing coronary occlusion. 
Extending CT coverage from the skull base through the thorax may be useful in other patients presenting with neurological deficit, but the now 'quadruple rule-out' (including carotid imaging) likewise carries the attendant risks of increased exposure to both radiation and contrast. Anxieties for the patient and physician do not stop there. As the imaging net is cast wider, the likelihood of picking up other coincidental pathology, often endocrine 'incidentalomas', increases. Although the term was coined 30 years ago, the further investigation, management and monitoring of these patients, asymptomatic from relatively common coincidental findings, is still hotly debated. ${ }^{4}$ The financial implications of the various follow- up protocols have not gone unnoticed by increasingly cash-strapped healthcare providers.

Where does this leave us? In a recent, thought-provoking editorial on coronary imaging, Wood suggests that desirable tests lead to decision-making that benefits the patient by improving outcomes. ${ }^{5}$ Perhaps, whatever diagnostic strategy we may favour, be it the rod or the net, we need to remind ourselves of this paradigm in our daily practice. An awareness and understanding of the potential risks, limitations and, indeed, consequences of medical investigation have never been more important to patients and the profession alike.

\section{REFERENCES}

I Thavendiranathan P, Bagai A, Ebidia AR et al. Do blood tests cause anemia in hospitalized patients? The effect of diagnostic phlebotomy on hemoglobin and hematocrit levels. J Gen Intern Med 2005; 20:520-4. doi:I0.1 III/j.I525-I497.2005.0094.x

2 Sanchis J, Bodí V, Llácer Á et al. Risk stratification of patients with acute chest pain and normal troponin concentrations. Heart 2005; 9|:I0|3-8. doi:I0.II36/hrt.2004.04I673

3 Takakuwa KM, Halpern EJ. Evaluation of a 'triple rule-out' coronary CT angiography protocol: use of 64-section CT in lowto-moderate risk emergency department patients suspected of having acute coronary syndrome. Radiology 2008; 248:438-46. doi: I0. I |48/radiol.2482072169

4 Gough J, Scott-Coombes D, Palazzo FF. Thyroid incidentaloma: an evidence-based assessment of management strategy. World J Surg 2008; 32:1264-8. doi:10.1007/s00268-008-9503-2

5 Wood WC. Non-invasive anatomy and physiology: the new 'gold standard'? Heart 20 I0; 96:4 I I-2. doi:I0.I I36/hrt.2009.18373 I

\section{MARK YOUR DIARY 50TH ST ANDREW'S DAY FESTIVAL SYMPOSIUM 2-3 December 2010 at the RCPE}

The RCPE held its first St Andrew's Day Festival Symposium in December 196I. This year, the College will be making the 50th symposium a celebration of medical advances and will include a historical perspective in its flagship event. There will be lots of practical 'how do I manage' sessions and the following topics will be included in the symposium: cardiology, gastroenterology, infectious diseases, neurology, palliative medicine, renal medicine and respiratory medicine. Please put the date in your diary. Further details will follow in due course. 